\title{
AVALIAÇÃO DA INFLUÊNCIA DE CONDIÇÕES METEOROLÓGICAS NA OCORRÊNCIA E MANUTENÇÃO DE UM EPISÓDIO PROLONGADO COM ALTAS CONCENTRAÇÕES DE OZÔNIO SOBRE A REGIÃO METROPOLITANA DE SÃO PAULO
}

\author{
VANESSA SILVEIRA BARRETO CARVALHO ${ }^{1}$, EDMILSON DIAS DE FREITAS ${ }^{2}$, CAROLINE \\ ROSARIO MAZZOLI², MARIA DE FÁTIMA ANDRADE ${ }^{2}$
}

\author{
${ }^{1}$ Instituto de Recursos Naturais, Universidade Federal de Itajubá, Itajubá, MG, Brasil \\ ${ }^{2}$ Instituto de Astronomia, Geofísica e Ciências Atmosféricas, Universidade de São Paulo, São Paulo, SP, \\ Brasil
}

vanessa.silveira@gmail.com; efreitas@model.iag.usp.br; carol@model.iag.usp.br; mftandra@model.iag.usp.br

Recebido Maio de 2010 - Aceito Maio 2012

\begin{abstract}
RESUMO
Na Região Metropolitana de São Paulo, a rede de monitoramento da qualidade do ar registra frequentemente valores de concentração de ozônio superiores aos estabelecidos pela legislação em vigor no Brasil. Em algumas ocasiões, condições meteorológicas favoráveis ao processo de formação de ozônio possibilitam a ocorrência de altas concentrações de ozônio durante diversos dias consecutivos na região. A influência de condições meteorológicas no episódio de poluição, registrado entre os dias de 24 de fevereiro e 05 de março de 2003, foi avaliada através de simulações numéricas com o modelo SPM-BRAMS. Estudos observacionais mostram que a ocorrência de ventos fracos, principalmente, durante o período noturno e durante as manhãs, favorece a formação de altas concentrações de ozônio sobre a região. O horário de entrada e a atuação da brisa marítima são fundamentais para o transporte do ozônio e de seus precursores. Essas condições foram bem simuladas pelo modelo. Para os dias mais críticos, quando diversas estações de monitoramento registraram a ocorrência de violações ao padrão nacional de qualidade do ar, o modelo simulou adequadamente as condições atmosféricas determinantes para a manutenção de valores elevados nas concentrações de ozônio, sem a necessidade de nenhum procedimento artificial ao longo das integrações.
\end{abstract}

Palavras-Chave: Poluição atmosférica, Problemas urbanos, SPM-BRAMS.

\begin{abstract}
EVALUATION OF THE METEOROLOGICAL CONDITIONS INFLUENCE ON THE OCCURRENCE AND MAINTENANCE OF A MULTIDAY EPISODE WITH HIGH OZONE CONCENTRATIONS OVER THE METROPOLITAN AREA OF SÃO PAULO

In the Metropolitan Area of Sao Paulo, air quality monitoring network often register ozone concentration values higher than those established by Brazilian legislation. In some occasions, weather conditions favorable to ozone formation allow the occurrence of high concentration values for several consecutive days in the area. The influence of meteorological conditions on the air pollution episode registered between February 24 and March 05, 2003 was assessed through numerical simulations with the SPM-BRAMS model. Observational studies show that the occurrence of calm winds, especially during the night and morning, favor the formation of high ozone concentration values over the region. The penetration time and the influence of the sea breeze are essential for the transport of ozone and its precursors. These conditions were well simulated by the model. For the most critical days, when several monitoring stations recorded the occurrence of violations to the ozone National Air Quality Standard, the model simulated properly the atmospheric conditions needed for the maintenance of high ozone concentration values, without any artificial procedure during the integrations.
\end{abstract}

Keywords: Atmospheric Pollution, Urban Problems, SPM-BRAMS 


\section{INTRODUÇÃO}

Mais de 19 milhões de pessoas residem em trinta e nove municípios que compõem a Região Metropolitana de São Paulo (RMSP). Na área urbana, com aproximadamente $8000 \mathrm{~km}^{2}$, circulam mais de 7 milhões de veículos. Estima-se que a frota veicular seja responsável por $97 \%$ das emissões de monóxido de carbono (CO) e hidrocarbonetos (HC), 96\% de óxidos de nitrogênio $\left(\mathrm{NO}_{\mathrm{X}}\right), 42 \%$ de dióxido de enxofre $\left(\mathrm{SO}_{2}\right)$ e $40 \%$ de material particulado (PM) (CETESB, 2008).

As concentrações de ozônio $\left(\mathrm{O}_{3}\right)$ monitoradas na RMSP, frequentemente, apresentam violações ao padrão nacional de qualidade do ar (PNQA) (Martins et al., 2004; Freitas et al., 2005; Martins e Andrade, 2008; Sánchez-Ccoyllo et al., 2006). De acordo com os relatórios anuais de qualidade do ar produzidos pela Companhia de Tecnologia de Saneamento Ambiental do Estado de São Paulo - CETESB (CETESB, 2003; CETESB, 2008), o número médio de dias com ultrapassagens ao padrão de 1 hora $\left(160 \mu \mathrm{g} \mathrm{m}^{-3}\right)$ é superior a 68 dias por ano, considerando o período entre os anos de 1999 e 2007. Entre 2002 e 2006, foi registrada pela rede de monitoramento em operação na RMSP, uma diminuição no número de dias em que as concentrações de ozônio foram superiores ao PNQA. Contudo, em 2007 foi registrado novamente um aumento nesse número, que atingiu o valor mais alto desde 2004 (observar Tabela 1). O comportamento sazonal destas, ilustrado na Figura 1, revela a ocorrência de violações durante todo o ano, principalmente, do final do inverno até o início do outono (ago-abr). Os menores valores são registrados durante o inverno. Climatologicamente, o inverno é caracterizado como a estação seca, com ventos

Tabela 1 - Número de dias com concentrações de ozônio superiores ao determinado pelo PNQA registrados no período entre 1999 e 2007 na RMSP.

\begin{tabular}{cc}
\hline Ano & $\begin{array}{c}\mathrm{N}^{\circ} \text { de dias de } \\
\text { ultrapassagens do PNQA }\end{array}$ \\
\hline 1999 & 80 \\
2000 & 67 \\
2001 & 78 \\
2002 & 82 \\
2003 & 77 \\
2004 & 63 \\
2005 & 51 \\
2006 & 46 \\
2007 & 72 \\
\hline
\end{tabular}

calmos e com atuação predominante de um sistema de alta pressão sobre o continente (Ulke e Andrade, 2001; Martins e Andrade, 2008; Sánchez-Ccoyllo et al., 2006).

A RMSP está localizada em um platô a aproximadamente $720 \mathrm{~m}$ acima do nível do mar e a $60 \mathrm{~km}$ de distância da costa do Atlântico Sul. A topografia da região é altamente complexa com a Serra da Mantiqueira a noroeste e com a presença da Serra do Mar a sul e sudeste (Silva Dias e Machado, 1997; SánchezCcoyllo e Andrade, 2002; Freitas et al., 2005). Por conta desta configuração, as circulações de mesoescala possuem um importante papel no transporte de poluentes na região durante todo o ano (Silva Dias et al., 1995; Silva Dias e Machado, 1997).

Diversos estudos avaliaram a formação de ozônio na RMSP por meio da utilização de modelos numéricos (Andrade et al., 2004; Freitas et al., 2005; Martins et al., 2006; Martins e Andrade, 2008, Sánchez-Ccoyllo et al., 2006; Vivanco e Andrade, 2008). A influência de algumas parametrizações de turbulência e de variáveis meteorológicas nas concentrações de ozônio para episódios de curto período, simulados através do modelo CIT para a RMSP, foi discutida por Ulke e Andrade (2001) e Sánchez-Ccolyllo et al. (2006), respectivamente. Contudo, não há na literatura registro de estudos focando o papel das circulações de mesoescala durante a ocorrência de episódios prolongados com altas concentrações de ozônio na RMSP. No presente estudo, o modelo de mesoescala BRAMS, acoplado ao módulo fotoquímico simplificado (SPM, Simple Photochemical Module), foi utilizado para a análise da influência destas circulações na formação e manutenção de um episódio prolongado com altas concentrações de ozônio sobre a RMSP. Dessa forma, foram analisadas as condições meteorológicas prognosticadas pelo BRAMS, além das concentrações de ozônio e de seus precursores geradas por este. A avaliação da

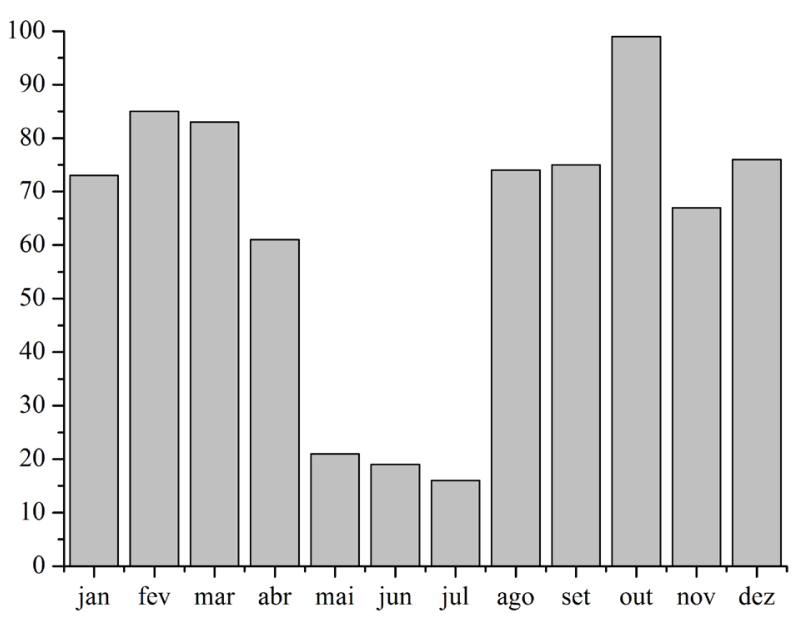

Figura 1 - Distribuição do número de dias totais com ultrapassagens ao PNQA estabelecido para o ozônio registrados em estações de monitoramento na RMSP no período de 1996 até 2006. 
influência destas condições nos resultados de concentração de ozônio obtidos com o SPM foi o principal alvo deste estudo.

\section{MATERIAL E MÉTODOS}

\subsection{Período de estudo}

O período considerado por este estudo, entre os dias 24 de fevereiro e 05 de março de 2003, apresentou, durante vários dias consecutivos e em várias estações de qualidade de ar que compõem a rede de monitoramento da CETESB, níveis de concentração de ozônio superiores ao PNQA estabelecido para o ozônio, igual a $160 \mu \mathrm{gm}^{-3}$ (CETESB, 2008). As máximas concentrações diárias de ozônio registradas em todas as estações de qualidade do ar disponíveis na RMSP nos períodos de interesse podem ser observadas na Tabela 2. A partir dessa, é possível verificar a ocorrência de valores bem acima do definido pela legislação vigente, como seguro para a saúde e o bem estar da população.

Durante esse período, toda a região sudeste do Brasil esteve sobre a influência da alta subtropical do Atlântico Sul, deslocada para oeste de sua posição climatológica. A atuação desse sistema também foi verificada por Abreu (1985) em outros episódios com altas concentrações de poluentes registrados em São Paulo. A predominância dos ventos em escala sinótica durante o período foi de sudeste, mas com a ocorrência de ventos de NE, principalmente, entre os dias 28 de fevereiro e 03 de março. Associado a este sistema, além de condições com ventos fracos, foram observadas condições de céu claro ou com pouca nebulosidade, alta incidência de radiação solar e ausência de precipitação até o dia 05 de março, quando uma frente fria atingiu o Estado de São Paulo. Os dados provenientes do METAR (www.redemet.aer.mil.br), produzido no aeroporto de Guarulhos (Figura 2), confirmaram a predominância de condições de céu-claro ou pouca cobertura de nuvens na maior parte do período. No período diurno, entre 06 e 18 Hora Local, o número de horas em que a cobertura de nuvens foi de no máximo $2 / 8$ foi igual a $67 \%$. Vale ressaltar que a atuação de sistemas de alta pressão, geralmente associados a condições de céu-claro, altas temperaturas e ventos fracos, foi relatada por diversos estudos como favorável à ocorrência de altos índices de ozônio na superfície (Comrie e Yarnal, 1992; Comrie, 1996; Sillman, 1999; Vukovich et al., 1999; Diem e Comrie, 2001; Jenkin et al., 2002; Galvez, 2007).

\subsection{Descrição e configuração do modelo}

O modelo atmosférico BRAMS é a versão brasileira do Regional Atmospheric Modeling System (RAMS, Cotton et al., 2003), desenvolvido inicialmente pela Universidade do Estado do Colorado - EUA e pela Mission Research Coorporation/Aster Inc. (MRC/*ASTER) para simular circulações atmosféricas que

Tabela 2 - Máximas concentrações de ozônio, em $\mu$ g/m3, registradas na RMSP durante o período de 24/02/2003 a 05/03/2003.

\begin{tabular}{lcccccccccc}
\hline \multicolumn{1}{c}{ Data } & & & & & & & & & & \\
Estações & $24 / 02$ & $25 / 02$ & $26 / 02$ & $27 / 02$ & $28 / 02$ & $01 / 03$ & $02 / 03$ & $03 / 03$ & $04 / 03$ & $05 / 03$ \\
\hline Diadema & 252,62 & 201,51 & 134,64 & 161,38 & 193,91 & 212,18 & 153,39 & 173,93 & 239,54 & 130,10 \\
Ibirapuera & 232,78 & 180,44 & 170,39 & 208,41 & 256,56 & 235,93 & 162,84 & 163,52 & 279,08 & 172,85 \\
Mauá & 225,98 & 238,22 & 154,87 & 140,73 & 178,64 & 212,10 & 262,74 & 178,66 & 208,91 & 108,43 \\
Moóca & 203,85 & 148,59 & 177,43 & 175,52 & 183,76 & 200,35 & 141,79 & 151,18 & 227,71 & 126,29 \\
Parque Dom Pedro & 171,74 & 118,64 & 155,71 & 152,12 & 178,96 & 174,33 & 121,40 & 110,57 & 232,00 & 107,94 \\
Pinheiros & 131,64 & 108,00 & 101,58 & 151,57 & 204,87 & 131,60 & 122,81 & 106,94 & 235,59 & 110,81 \\
São Miguel Paulista & 194,22 & 216,64 & 156,87 & 126,25 & 138,66 & 116,44 & 135,53 & 118,86 & 168,21 & 138,09 \\
Santo André & 246,66 & 265,76 & 132,52 & 141,68 & 180,10 & 205,37 & 224,37 & 175,75 & 257,56 & 112,83 \\
Santana & 187,02 & 157,11 & 222,08 & 178,70 & 217,63 & 193,62 & 143,32 & 151,41 & 264,39 & 155,63 \\
São Caetano do Sul & 272,55 & & 140,95 & 159,40 & 195,55 & 242,79 & 219,73 & 182,90 & 267,24 & 129,84 \\
Santo Amaro & 244,05 & 202,57 & 162,78 & 233,13 & 278,77 & 238,19 & 180,81 & 212,06 & 218,23 & 185,09 \\
\hline
\end{tabular}




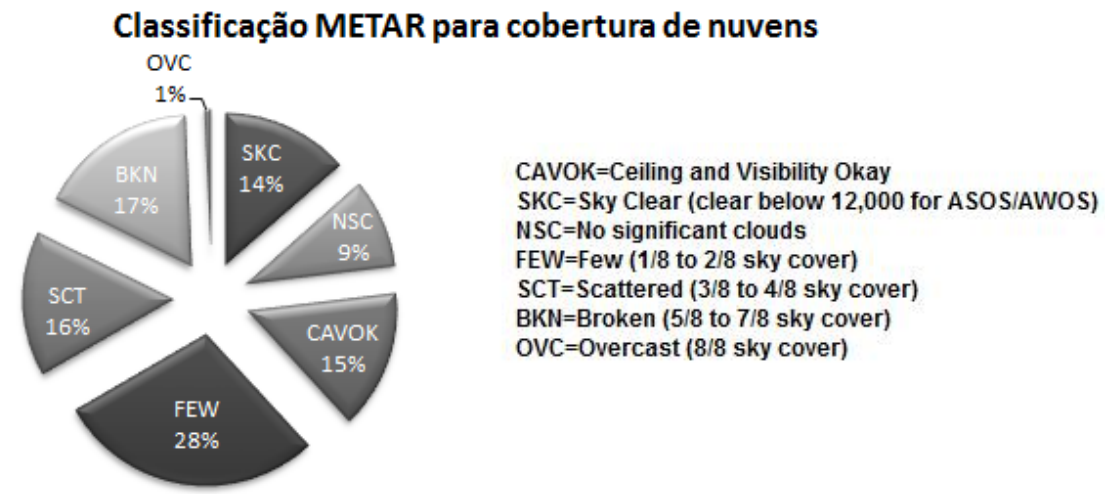

Figura 2 - Frequência de ocorrência das classes de cobertura de nuvens utilizadas em mensagens METAR para os horários entre 06 e 18 Horal Local para cada dia no período entre 24 de fevereiro de 2003 e 06 de março de 2003.

envolvem desde a micro até a grande escala, sendo a modelagem dos fenômenos de mesoescala sua mais freqüente aplicação. Além de representar fisicamente os processos radiativos, transportes convectivos associados às nuvens e à turbulência seca, o modelo revela-se adequado para a avaliação dos efeitos de fatores locais, tais como a topografia, a presença de corpos d'água de grandes dimensões e áreas urbanas, aspectos de interesse do presente estudo. O processo de formação de ozônio também é considerado pelo modelo através do Módulo Fotoquímico Simplificado - SPM. O acoplamento e a validação deste módulo, que contém apenas 15 reações e foi desenvolvido visando a realização de previsões das concentrações de ozônio para a RMSP em caráter operacional, é descrito por Freitas et al. (2005).

As condições iniciais e de fronteira foram obtidas a partir das análises do Global Forecast System (GFS) do National Centers for Environmental Prediction (NCEP), disponíveis em intervalos de 6 horas. As simulações foram iniciadas 24 horas antes do período de interesse, sendo o primeiro dia descartado das análises para minimizar o efeito de spin-up. Duas grades aninhadas, centradas em $23,55^{\circ} \mathrm{S}$ e $46,47^{\circ} \mathrm{W}$, com espaçamento horizontal de grade de 16 e $4 \mathrm{~km}$, respectivamente, e 33 níveis verticais foram considerados na simulação.

Em relação aos dados de emissão, para a RMSP, apenas um valor anual total de emissão veicular e industrial é disponibilizado pela CETESB. São fornecidos por este inventário emissões de $\mathrm{CO}, \mathrm{NO}_{\mathrm{X}}, \mathrm{PM}_{10}, \mathrm{SO}_{2}$ e HC. Conforme apresentado em Freitas et al. (2005), a distribuição espacial das emissões sobre a RMSP é realizada com base no tipo de ocupação urbana, considerando duas áreas principais, definidas em Freitas (2003). A primeira, localizada sobre o centro da RMSP, é responsável por 70\% das emissões veiculares e a segunda, uma área menos urbanizada (em relação à primeira), responsável pelos $30 \%$ restantes. No caso das emissões industriais, o contrário é considerado, uma vez que a maior contribuição de fontes fixas, tais como indústrias, comércio, queima de resíduos, ocorre nas áreas que circundam o centro empresarial.

\section{RESULTADOS E DISCUSSÃO}

Para o período simulado, o modelo BRAMS apresentou resultados coerentes com aqueles observados, com predominância de condições de céu claro. No final da tarde e início da noite do dia 03 de março, os resultados indicaram a presença de nebulosidade sobre todo o leste da RMSP com ocorrência de precipitação sobre o litoral do estado (Figura 3a). No dia 04 de março, como ilustrado na Figura 3b, durante a tarde, também foi verificada precipitação e nebulosidade, principalmente, sobre o sudoeste e norte do domínio. A partir da tarde do dia 05 também houve um aumento da fração de cobertura de nuvens em praticamente toda a grade considerada. Esse resultado apresenta coerência com o registrado no METAR do aeroporto de Guarulhos como pode ser observado na Figura 4.

As observações meteorológicas produzidas através do METAR também revelaram a ocorrência de altas temperaturas durante todo o período. A média das máximas ficou em torno de $32{ }^{\circ} \mathrm{C}$. A velocidade média dos ventos registrados durante o período no aeroporto de Guarulhos revelou valores ligeiramente acima dos $2 \mathrm{~m} \cdot \mathrm{s}^{-1}$. Durante todo o período simulado, o modelo também apresentou resultados condizentes com as observações, como apresentados em Carvalho (2010). Foram identificados altos valores de temperatura e baixa umidade relativa durante o final da manhã e todas as tardes (Figura 5a), além de ventos fracos, principalmente durante as madrugadas e as manhãs (Figura 5b).

$\mathrm{O}$ maior número de estações com registros superiores ao limite estabelecido pelo CONAMA (Conselho Nacional do Meio Ambiente) ocorreu nos dias 24 e 28 de fevereiro e 01 e 04 de março. A concentração média de ozônio registrada na RMSP, durante esse período, pode ser observada na Figura 6. Os valores apresentados nesta foram obtidos a partir dos dados de onze (11) estações de monitoramento alocadas na região. $\mathrm{Na}$ maior parte deste período foram identificadas características similares, em que o padrão sinótico de grande escala com ventos fracos favoreceu a propagação de circulações de mesoescala. 

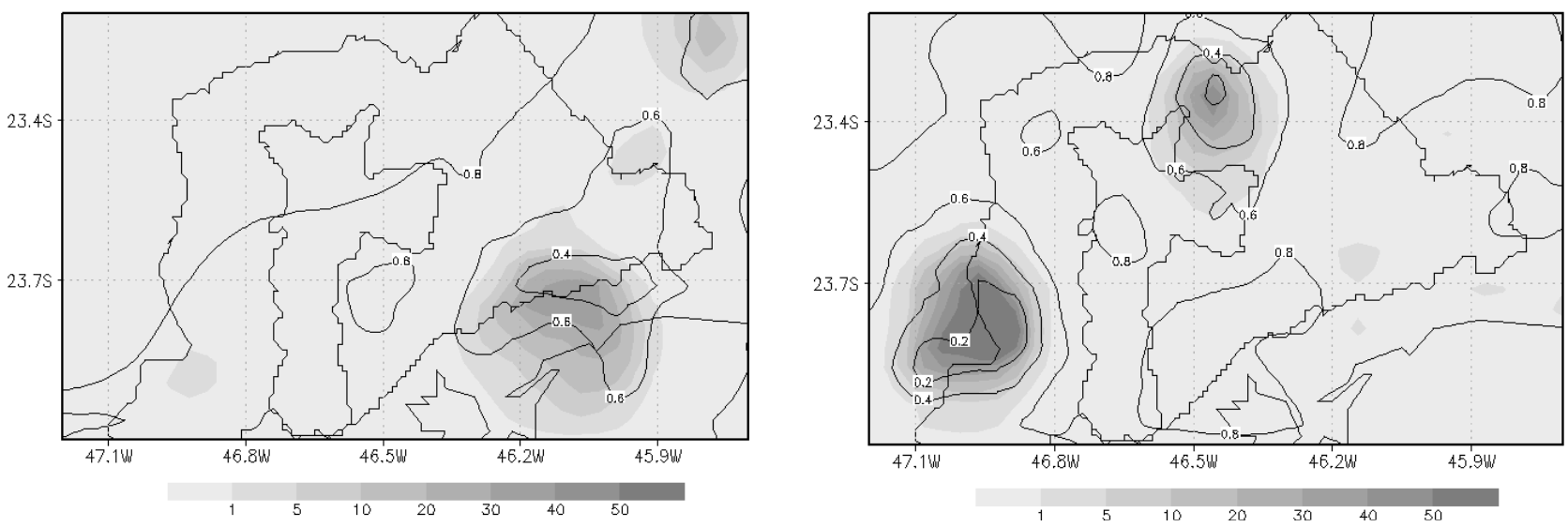

Figura 3 - Fração de cobertura de nuvens (contornos) e precipitação (sombreado), em mm/hora, simuladas pelo modelo BRAMS para os dias (a) $03 / 03 / 2003$ às $20 \mathrm{Z} \mathrm{e} \mathrm{(b)} \mathrm{04/03/2003} \mathrm{às} 16 \mathrm{Z}$.

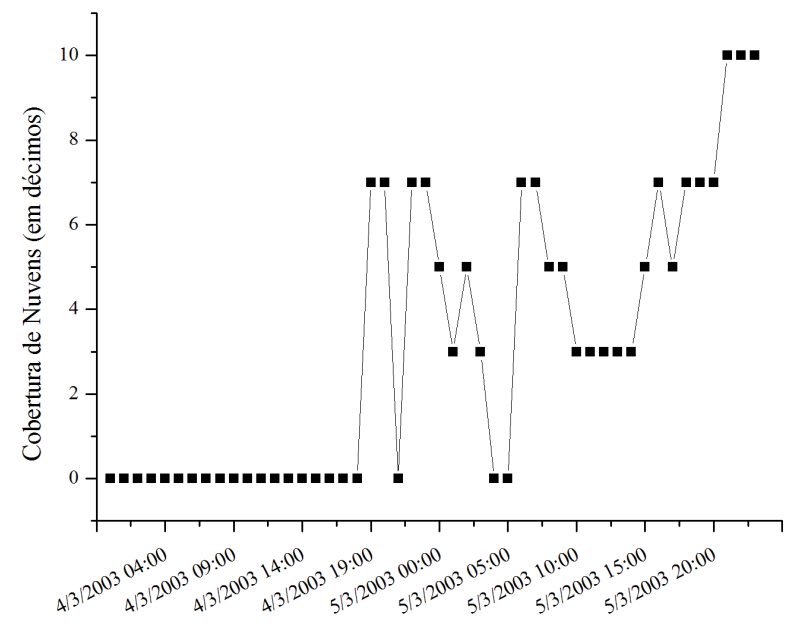

Figura 4 - Registros de cobertura de nuvens (em décimos) obtidos em mensagens METAR registradas no Aeroporto de Guarulhos, entre os dias 04 e 05 de março de 2003.

Em geral, foram verificados ventos fracos, principalmente, durante a madrugada e manhã com intensificação no início da tarde dos ventos de SE, associados à penetração da brisa marítima. $\mathrm{O}$ horário em que a frente de brisa atingiu o centro da grade variou um pouco de acordo com o dia. Vale ressaltar que o centro da grade (ponto com latitude $23,6^{\circ} \mathrm{S}$ e longitude $46,4^{\circ} \mathrm{W}$ ) está localizado a $60 \mathrm{~km}$ da linha de costa. O horário no qual a brisa marítima atingiu esse ponto foi determinado através do aumento da velocidade dos ventos e do aumento da razão de mistura. A atuação da brisa marítima auxiliou no transporte das concentrações de ozônio e de seus precursores como será apresentado adiante.

No dia 24, por exemplo, maiores níveis de concentração de ozônio começaram a ser observados na RMSP às 10 horas da manhã (Figura 6). A maior intensidade da pluma foi simulada pelo modelo entre meio dia e uma hora da tarde. Por cinco horas após o seu início, a pluma não se deslocou, mantendo-se centrada, principalmente, sobre parte dos municípios de São Paulo e Osasco. Durante esse período, os ventos simulados sobre a região foram bem fracos. Apenas às 16 horas, com a entrada da brisa marítima, a pluma começa a se deslocar em direção ao noroeste da RMSP. A evolução do campo de vento e das concentrações de ozônio simuladas a partir das 10 horas (com intervalo de 2 horas) pode ser observada na Figura 7.

Outro aspecto interessante verificado nos resultados do modelo referentes ao dia 24 de fevereiro foi a amplitude dos picos de concentração de $\mathrm{NO}_{\mathrm{X}}$, em média menor do que nos demais dias do período. Os valores máximos simulados ficaram em torno de $50 \mu \mathrm{g} . \mathrm{m}^{-3}$, durante a manhã, e $40 \mu \mathrm{g} . \mathrm{m}^{-3}$, no período noturno, como pode ser observado na Figura 8. Em geral, considerando todo o período, o contrário foi identificado na maioria dos dias simulados, com maiores valores durante a noite. Esse comportamento é condizente com aquele registrado pela rede de monitoramento da CETESB.

Em relação às variáveis meteorológicas simuladas pelo modelo, além das condições de ventos calmos, também foram simulados, durante o final da manhã e parte da tarde do dia 24 de fevereiro, altos valores de temperatura do ar e de radiação de onda curta incidente (superiores a $30{ }^{\circ} \mathrm{C}$ e 900 W. $\mathrm{m}^{-2}$, respectivamente) e baixos valores de umidade relativa (inferiores a $40 \%$ na maior parte do domínio). A altura da camada limite planetária (CLP) variou entre $250 \mathrm{~m}$ e $2250 \mathrm{~m}$ durante o dia. Durante a madrugada e a manhã do dia 24 , boa parte da grade apresentou valores inferiores a $500 \mathrm{~m}$. No final da manhã, a altura média da CLP na RMSP passou a aumentar, ficando em torno de $1000 \mathrm{~m}$. Ao final da tarde, os valores eram superiores a $1500 \mathrm{~m}$ em todo o domínio, exceto na faixa litorânea, onde foram simulados valores inferiores a $1000 \mathrm{~m}$.

No dia 25 de fevereiro, um grande núcleo com concentrações de ozônio, que caracterizam violações ao 


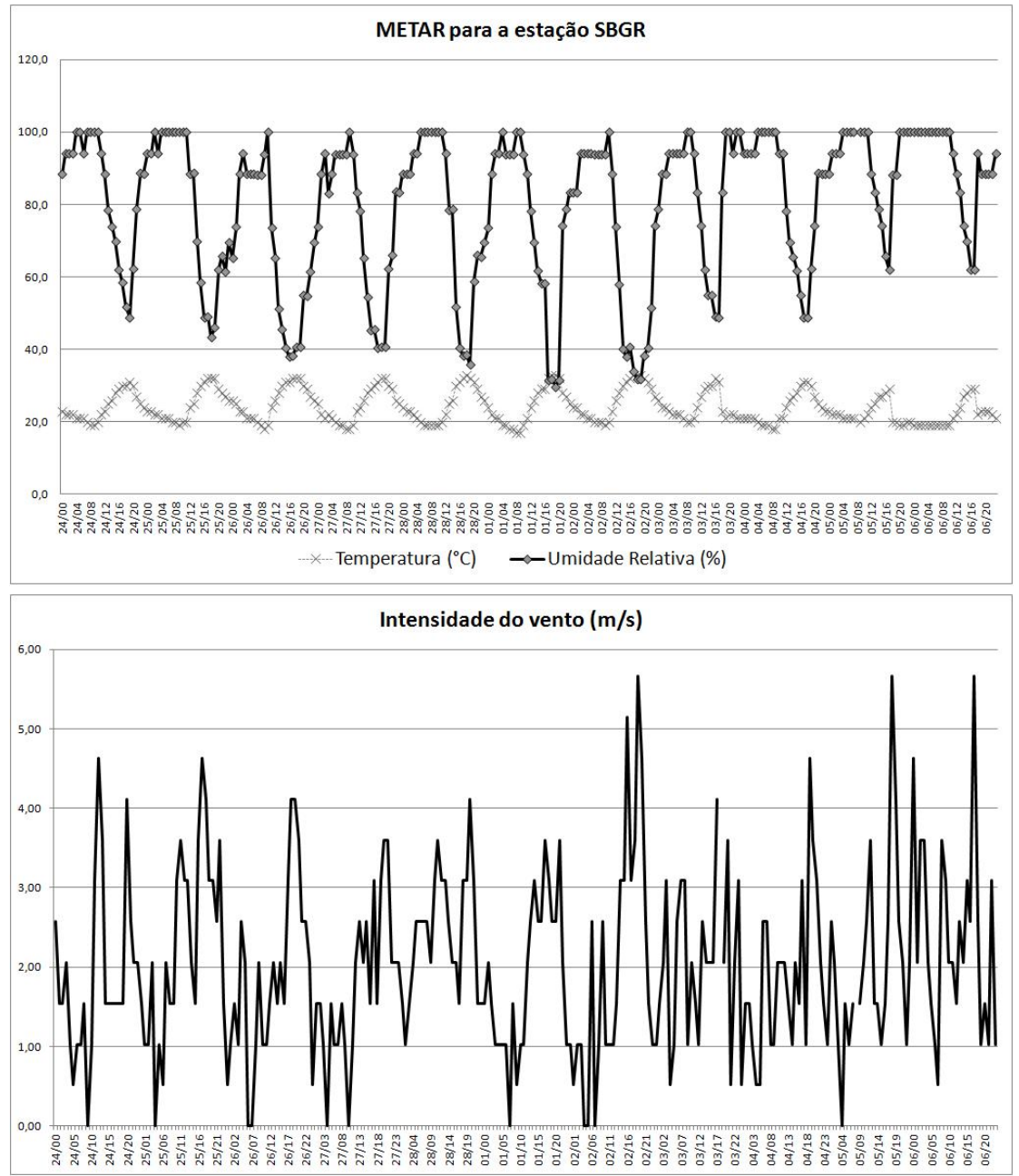

Figura 5 - Temperatura e Umidade relativa (a) e intensidade do vento (b) obtidos das mensagens METAR para a estação SBGR entre 24 de fevereiro e 06 de março de 2003.

PNQA, foi verificado sobre o centro da RMSP entre 15 e $19 \mathrm{Z}$ (observar Figura 9a). Apenas após as $20 \mathrm{Z}$, as concentrações começaram a diminuir e a pluma foi deslocada para NW com a entrada da frente de brisa. Nos dias 26 e 27, as concentrações de $\mathrm{O}_{3}$ simuladas pelo modelo foram menores e o centro da pluma esteve ligeiramente deslocado para noroeste (exemplo na Figura 9b). Uma característica comum nestes três dias foi a não dissipação por completo da pluma de $\mathrm{NO}_{\mathrm{X}}$, que permaneceu durante todo o dia sobre o centro da grade. Durante a madrugada, também foi observado na simulação que a pluma persistiu, contudo, com menor intensidade, sobre a região.

Durante os dias 28 de fevereiro e 01 de março características bem similares foram verificadas. Nesses dois dias a pluma de concentração de ozônio começou a surgir às 11 $\mathrm{Z}$, com máxima intensidade às $17 \mathrm{Z}$, quando maiores valores foram verificados em boa parte da RMSP e em parte do litoral sobre os municípios de Cubatão e Santos (observar Figura 10). A partir desse horário, o deslocamento da pluma para noroeste,

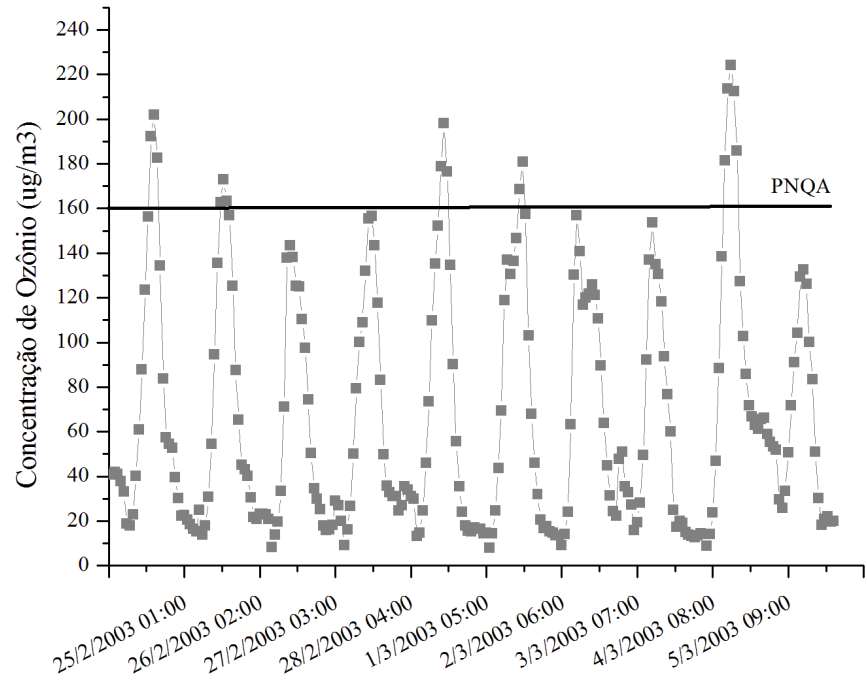

Figura 6 - Concentração Média de Ozônio na RMSP registrada no período entre 24 de fevereiro e 05 de março de 2003. 

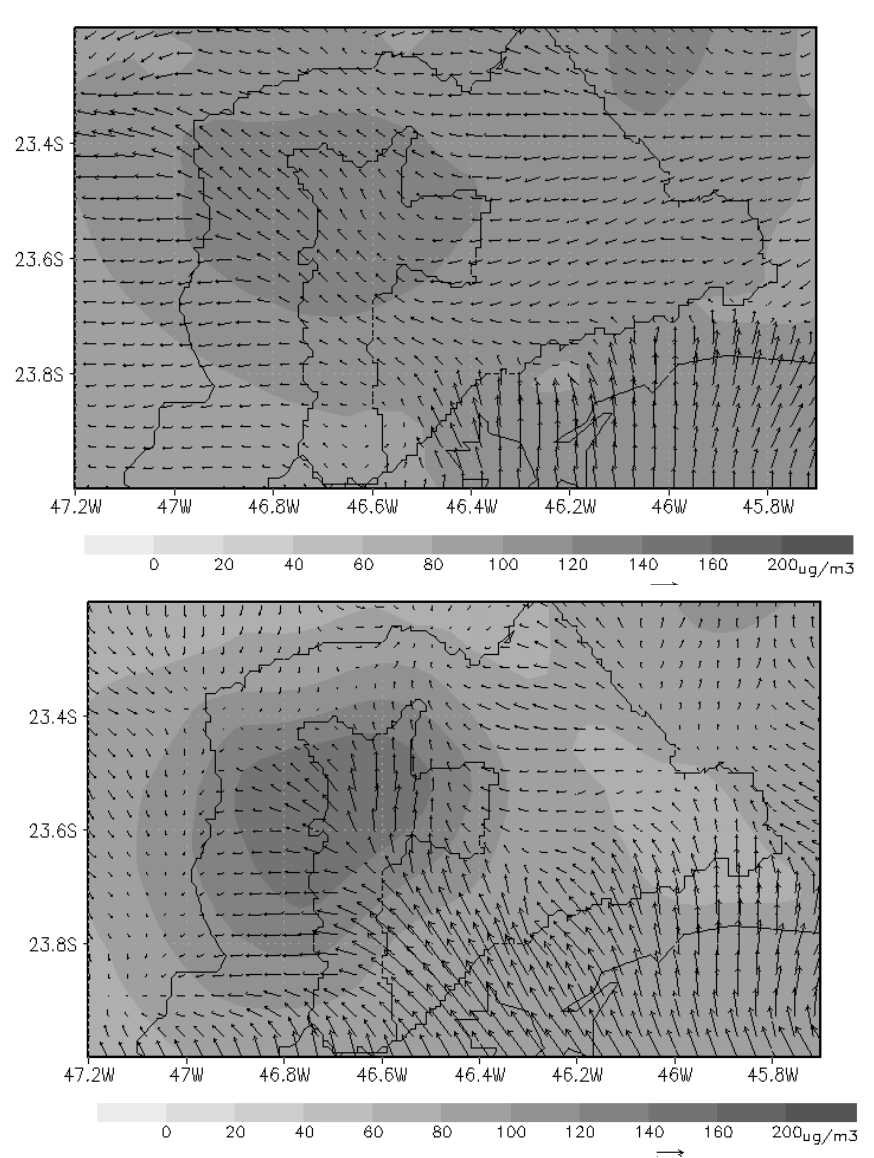
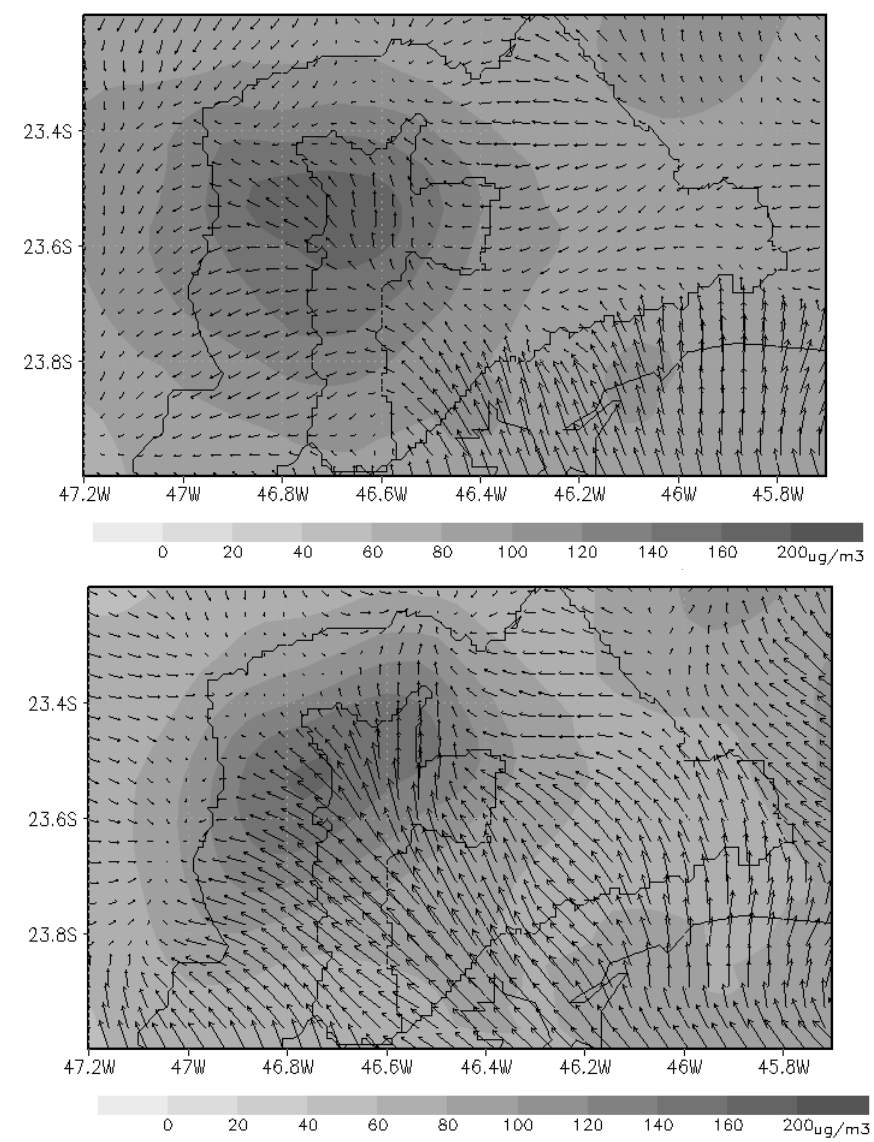

Figura 7 - Campo de Vento (m s-1) e de Concentração de Ozônio ( $\mu$ g.m-3) referente ao dia 24 de fevereiro (a) 12 Z, (b) 14 Z, (c) 16 Z e (d) 18 Z.
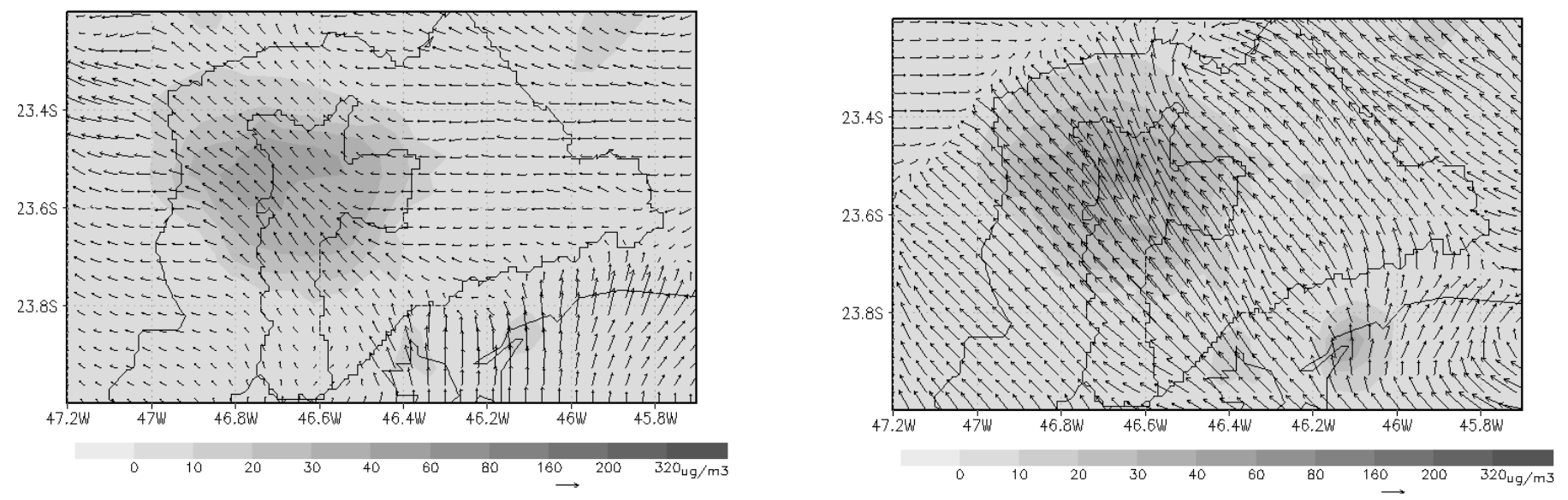

Figura 8 - Campo de Vento (m s-1) e de Concentração de NOX ( $\mu$ g.m-3) referente ao dia 24 de fevereiro (a) 11 Z e (b) 21 Z.

impulsionado pela entrada da brisa marítima, foi rápido.

Outro ponto em comum foi o acúmulo de $\mathrm{NO}_{X}$ simulado durante a madrugada. Em geral, a pluma de $\mathrm{NO}_{\mathrm{X}}$ se dissipa após o pico noturno. Contudo, tanto no dia 28 quanto no dia 01, durante a madrugada, a pluma não se dissipou e manteve-se sobre o centro-oeste da RMSP. Durante a manhã, com o aumento da emissão veicular, os níveis de $\mathrm{NO}_{\mathrm{X}}$ ultrapassam os 200 $\mu \mathrm{g} . \mathrm{m}^{-3}$, como pode ser visto na Figura 11. De modo similar ao ocorrido no dia 24 de fevereiro, as concentrações dos óxidos de nitrogênio foram superiores durante o dia.

As condições meteorológicas simuladas pelo modelo para os dias 28 de fevereiro e 01 de março revelaram a ausência 

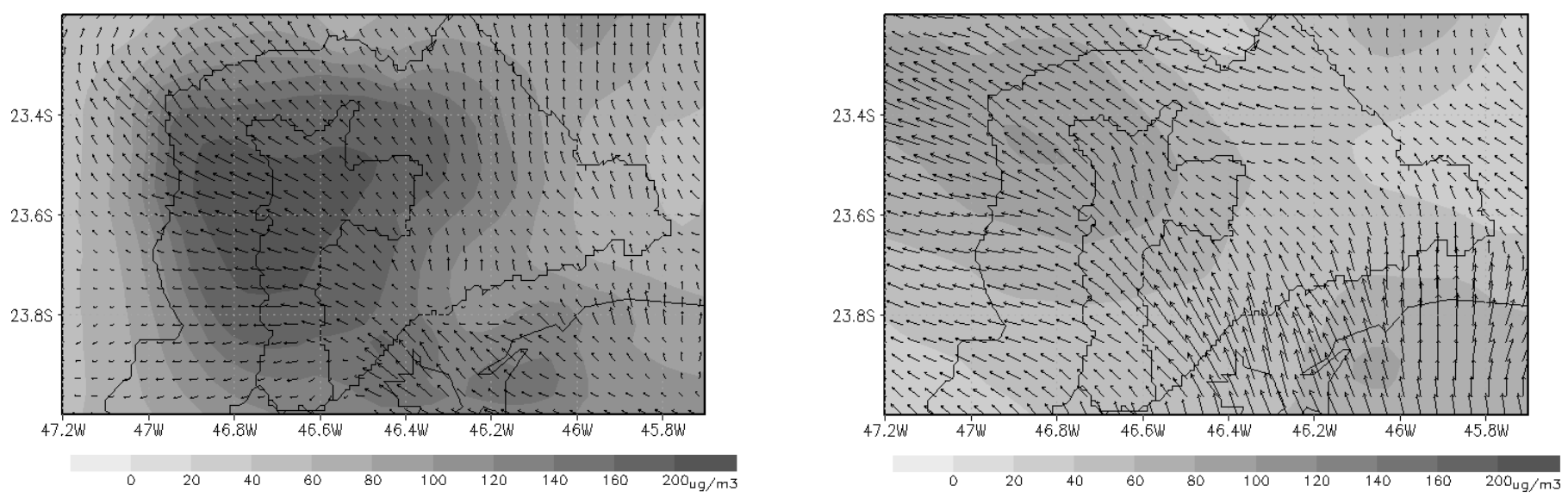

Figura 9 - Campo de Vento (m s-1) e de Concentração de Ozônio ( $\mu$ g.m-3) referente aos dias (a) 25 de fevereiro às $18 \mathrm{Z}$ e (b) 27 de fevereiro às $14 \mathrm{Z}$.
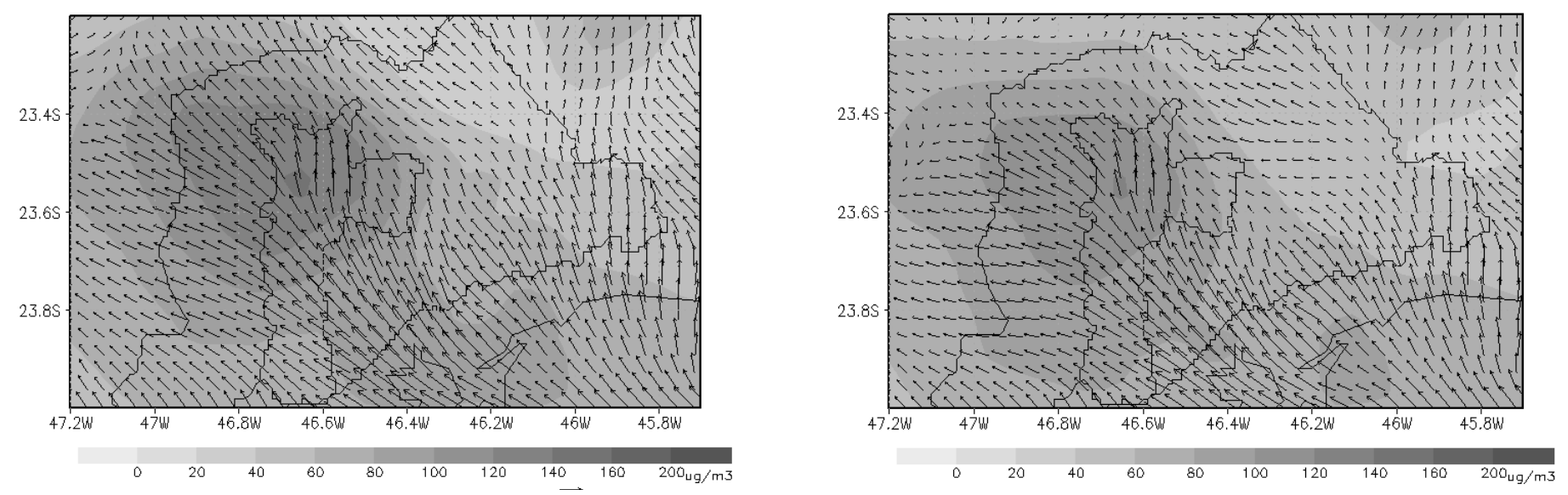

Figura 10 - Campo de Vento (m s-1) e de Concentração de Ozônio ( $\mu$ g.m-3) referente aos dias (a) 28 de fevereiro às $17 \mathrm{Z}$ e (b) 01 de março às $17 \mathrm{Z}$.
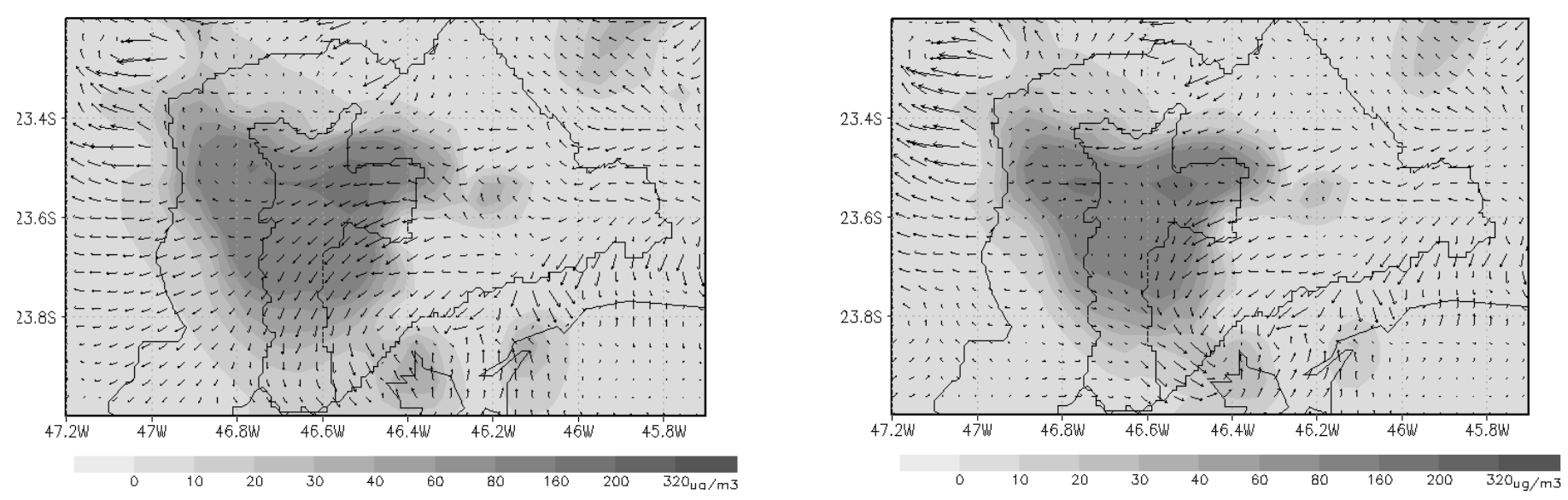

Figura 11 - Campo de Vento (m s-1) e de Concentração de NOX ( $\mu$ g.m-3) referente aos dias (a) 28 de fevereiro às $10 \mathrm{Z}$ e (b) 01 de março às $10 \mathrm{Z}$. 

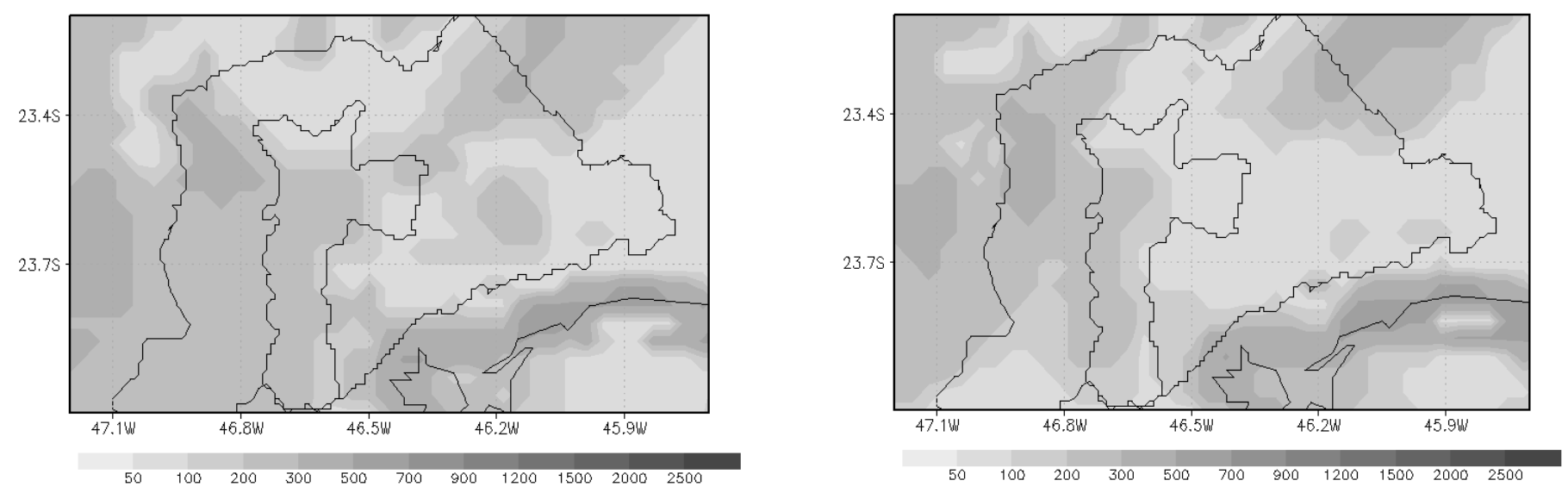

Figura 12 - Altura da CLP (m) referente aos dias (a) 28 de fevereiro às $06 \mathrm{Z} \mathrm{e} \mathrm{(b)} 01$ de março às $06 \mathrm{Z}$.

de nuvens e de precipitação em todo o domínio. A temperatura esteve acima dos $30^{\circ} \mathrm{C}$ e os valores de UR foram inferiores a $30 \%$ durante a tarde em ambos os dias. Em toda a RMSP os valores de altura da CLP (Figura 12) foram inferiores a $300 \mathrm{~m}$ durante toda a madrugada e início da manhã, o que pode ter contribuído para o acúmulo dos $\mathrm{NO}_{\mathrm{X}}$, assim como, de outros precursores. No final da manhã, conforme o esperado, a altura da CLP começa a aumentar, atingindo valores superiores a 2000 $\mathrm{m}$ em algumas áreas.

Durante a noite do dia 28 e madrugada do dia 01 também foi simulada a ocorrência de brisa terrestre, não muito intensa, em parte do litoral paulista. A partir das 21 horas (Hora Local), houve o enfraquecimento dos ventos sobre a linha de costa. No dia 28 de fevereiro, duas horas mais tarde, ocorreu a inversão dos ventos em alguns pontos do litoral (Figura 13), apesar dos ventos utilizados para a inicialização do modelo serem de SE. Assim como o identificado em Freitas (2003), não é possível observar a brisa terrestre sobre toda a faixa litorânea.

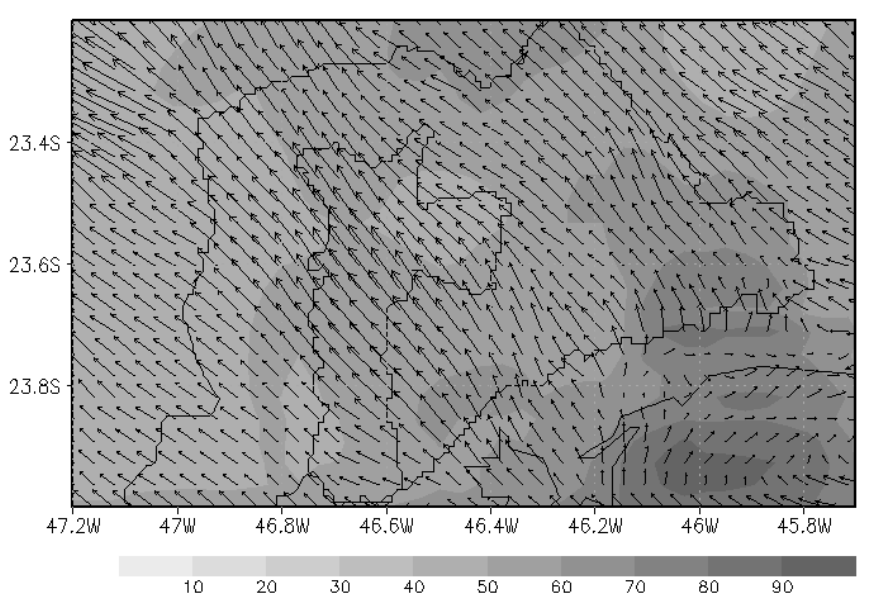

Figura 13 - Campo de Vento (m s-1) e de Umidade Relativa do ar (\%) referente ao dia 28 de fevereiro às $23 \mathrm{Z}$.
Nos dias 02 e 03 de março de 2003, os ventos de escala sinótica se intensificam ligeiramente e as concentrações de $\mathrm{O}_{3}$ simuladas pelo SPM-BRAMS, em geral, foram mais baixas do que as verificadas nos dias anteriores. Um aspecto interessante a ser destacado no dia 03 de março é a advecção da pluma de ozônio para sudeste durante parte da tarde. Apenas após as $18 \mathrm{Z}$, o núcleo com concentrações mais intensas passou a ser deslocado para noroeste, acompanhando a frente de brisa.

No dia 04 de março também foi identificada nas simulações a ocorrência de ventos ligeiramente mais intensos durante a madrugada e a manhã. A partir das 14 horas, também foi detectada a intensificação dos ventos associados com o aumento da atividade convectiva na região. Dois núcleos com concentrações de $\mathrm{O}_{3}$ e de $\mathrm{NO}_{\mathrm{X}}$ mais intensas, sobre o centro da RMSP e sobre parte do litoral, foram verificados (observar Figura 14). No caso das concentrações de $\mathrm{NO}_{\mathrm{X}}$ foi verificado que o núcleo permaneceu durante todo o dia centrado sobre a RMSP. Apenas a partir do final da noite, após o segundo pico de emissão de poluentes associado ao tráfego veicular, a pluma passou a ser transportada para NW.

A ocorrência de precipitação, por conta da aproximação de um sistema frontal, ainda que fraca, parece ter alterado a distribuição das concentrações de ozônio, ora mantendo os pontos de máxima intensidade no centro da grade (entre as células convectivas como pode ser observado na Figura 15a), ora os deslocando para fora destas (Figura 15b). Contudo, é importante ressaltar que o modelo não registrou queda nos valores de concentração de ozônio. Vale também destacar que, apesar de não representar o processo de deposição de poluentes de forma explicita, o modelo, em geral, consegue simular a redução nas concentrações de precursores e do próprio ozônio através da representação de fenômenos associados à turbulência e a dispersão destes poluentes.

No dia 05 de março, a maioria das estações da CETESB registrou queda nas concentrações de ozônio. 

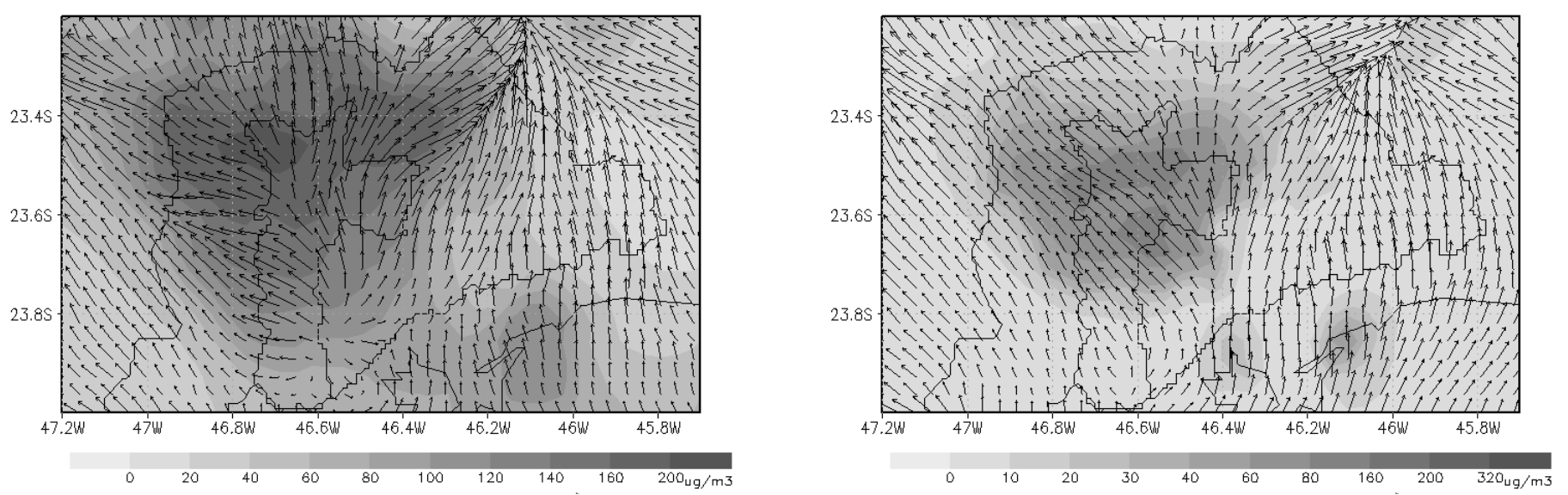

Figura 14 - Campo de Vento (m s-1) e de Concentração de (a) ozônio ( $\mu$ g.m-3) e (b) NOX ( $\mu$ g.m-3) referente ao dia 04 de março as 19 e 20 Z, respectivamente.
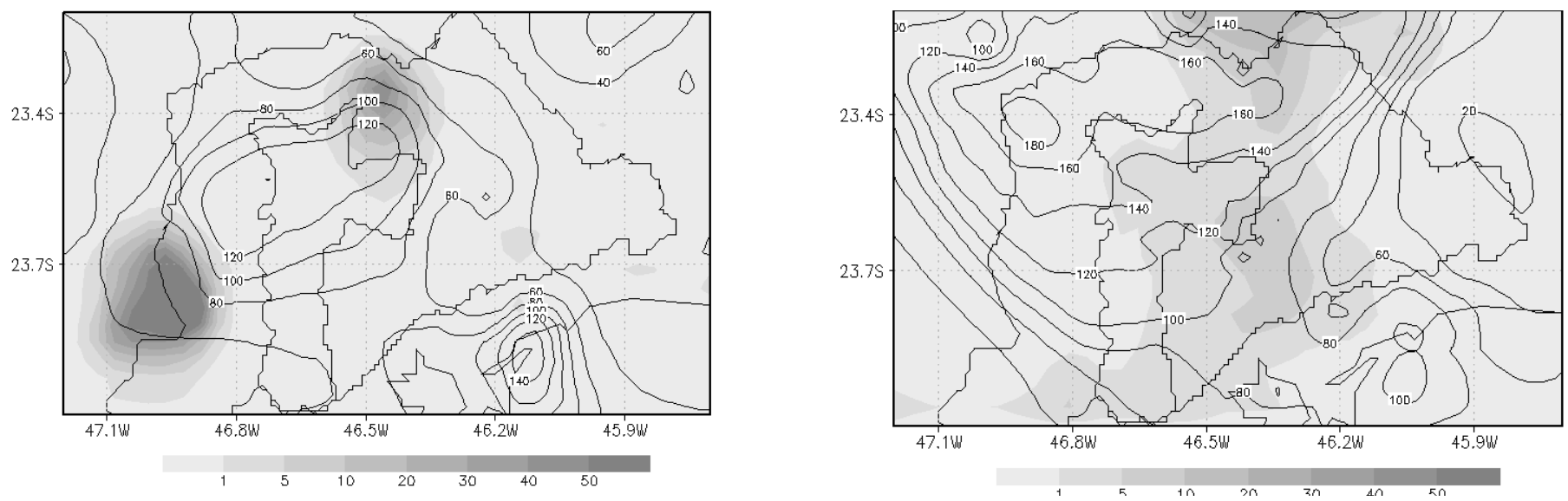

Figura 15 - Campo de precipitação (mm hora-1) e de Concentração de Ozônio ( $\mu$ g.m-3) referente ao dia 04 de março às (a)16 Z e (b) 20 Z.

Apenas as estações Ibirapuera e Santo Amaro registraram índices de qualidade do ar considerados inadequados. Observando os resultados da simulação é possível inferir que essa diminuição das concentrações de ozônio deve estar relacionada com o aumento da nebulosidade e conseqüente diminuição da radiação solar incidente, além, é claro, da ocorrência de precipitação que atuou, principalmente, na remoção de alguns precursores. O modelo SPM-BRAMS também registrou diminuição nas concentrações dos poluentes primários e secundários durante o dia 05 . Avaliando a evolução das concentrações de $\mathrm{NO}_{\mathrm{X}}$ foi possível detectar um leve aumento das concentrações no período da manhã (associado à intensificação do tráfego de veículos). Contudo, a pluma foi menos intensa do que normalmente seria em um dia de semana. O núcleo de concentrações dos óxidos de nitrogênio mais elevados permaneceu centrado sobre o município de São Paulo até às 20 horas. As máximas concentrações, inferiores a $80 \mu \mathrm{g} \mathrm{m}^{-3}$, foram simuladas mais cedo que o normalmente verificado, às 11 da manhã.

\section{CONCLUSÕES}

O presente estudo avaliou a influência das condições meteorológicas na ocorrência e manutenção de um episódio de alta concentração de ozônio por meio de simulações numéricas com o modelo SPM-BRAMS. Durante dez dias consecutivos, entre 24 de fevereiro e 05 de março de 2005, valores acima do estabelecido como PNQA foram registradas em diversas estações de qualidade do ar na RMSP. Os resultados da simulação com o modelo SPM-BRAMS indicaram que a ocorrência de ventos fracos durante no período noturno e durante a manhã contribuíram para o acúmulo de precursores sobre a RMSP. Os ventos fracos associados ao fluxo de grande escala favoreceram a atuação de circulações de mesoescala na região. A entrada da brisa marítima durante a tarde atuou, durante a maior parte do período, no transporte da pluma de concentração de ozônio para noroeste, reduzindo, desta maneira, as concentrações sobre a RMSP em horários que estas poderiam atingir valores ainda maiores. 
O modelo também simulou de maneira adequada para os dias com maior número de estações com violações ao PNQA condições de céu-claro, altos valores de temperatura do ar, de incidência de radiação solar de onda curta e baixos índices de umidade relativa. Os resultados simulados pelo modelo indicaram uma forte influência das condições meteorológicas no processo de formação e dispersão das concentrações de ozônio na RMSP, durante esse episódio com altas concentrações de ozônio. Os resultados também mostram a importância da utilização de modelos numéricos mais complexos, que integram equações e parametrizações relacionadas puramente aos processos atmosféricos, emissões de poluentes, reações químicas e outros processos relacionados à atividade humana, simultaneamente. Para a representação mais precisa das concentrações de ozônio é fundamental que as condições atmosféricas sejam bem representadas, o que foi relativamente observado durante as simulações realizadas.

\section{AGRADECIMENTOS}

Os autores agradecem à Fundação de Amparo a Pesquisa do Estado de São Paulo - FAPESP, pela bolsa de estudos da primeira autora (processo $\mathrm{n}^{\circ}$ 06/59546-9), e ao Conselho Nacional de Desenvolvimento Científico e Tecnológico - CNPq (processo $\mathrm{n}^{\circ} 477310 / 2008-7$ - Edital Universal, pelo auxílio financeiro concedido ao último autor).

\section{REFERÊNCIAS BIBLIOGRÁFICAS}

ABREU, M. L. Previsores sinótico-meteorológicos para a dispersão de poluentes na região da grande São Paulo. Dissertação de Mestrado do Instituto de Pesquisas Espaciais. São José dos Campos, INPE. INPE-3600- TDL/197. DRHDME. FRH/MET. pp. 131, 1985.

ANDRADE, M. F.; YNOUE, R. Y.; HARLEY, R.; MIGUEL, A. H. Air-Quality Model simulating photochemical formation of pollutants: the São Paulo Metropolitan Area, Brazil. International Journal of Environment and Pollution, Inglaterra, v. 22, n. 4, p. 460-475. 2004.

CARVALHO, V. S. B.. O impacto das megacidades na qualidade do ar em escala local e regional: os casos das Regiões Metropolitanas de São Paulo e do Rio de Janeiro. São Paulo, 2010, 237p. Tese de Doutorado do Departamento de Ciências Atmosféricas do Instituto de Astronomia, Geofísica e Ciências Atmosféricas da Universidade de São Paulo.

CETESB, 2003. Relatório de qualidade do ar no Estado de São Paulo 2002, São Paulo, 131 p.

CETESB, 2008. Relatório de qualidade do ar no Estado de São Paulo 2007, São Paulo, 284 p.
COTTON, W. R.; PIELKE, SR. R. A.; WALKO, R. L.; LISTON, G. E.; TREMBACK, C. J.; JIANG, H.; MCANELLY, R. L.; HARRINGTON, J. Y.; NICHOLLS, M. E.; CARRIO, G. G.; MCFADDEN, J. P. RAMS 2001: Current status and future directions. Meteorology and Atmospheric Physics, 82, 5-29. 2003.

COMRIE, A. C. An all-season synoptic climatology of air pollution in the US-Mexico border region. Professional Geographer, 48 (3), 237-251, 1996.

COMRIE, A. C.; YARNAL, B. Relationships between synopticscale atmospheric circulation and ozone concentrations in Metropolitan Pittsburgh, Pennsylvania. Atmospheric Environment, 26b (3), 301-312, 1992.

DIEM, J. E.; COMRIE, A. C. Air quality, climate, and policy: a case study of ozone pollution in Tucson, Arizona. Professional Geographer, 53 (4), 469-491, 2001.

FREITAS, E. D. Circulações locais em São Paulo e sua influência sobre a dispersão de poluentes. São Paulo, 2003, 156p. Tese de Doutoramento defendida no Departamento de Ciências Atmosféricas do Instituto de Astronomia, Geofísica e Ciências Atmosféricas da Universidade de São Paulo.

FREITAS, E. D., MARTINS, L. D., SILVA DIAS, P. L., ANDRADE, M. F. A simple photochemical module implemented in RAMS for tropospheric ozone concentration forecast in the Metropolitan Area of São Paulo - Brazil: coupling and validation. Atmospheric Environment 39(34), 6352-6361, 2005.

GALVEZ, O. Synoptic-scale transport of ozone into Southern Ontario. Atmospheric Environment, 41, 8579-8595, 2007.

JENKIN, M. E., DAVIES, T. J., STEDMAN, J. R. The origin and day-of-week dependence of photochemical ozone episodes in the UK. Atmospheric Environment, 36, 999-1013, 2002.

MARTINS, M. H. R. B.; ANAZIA, R.; GUARDANI, M. L. G.; LACAVA, C. I. V.; ROMANO, J.; SILVA, S. R. Evolution of air quality in the São Paulo Metropolitan Area and its relation with public policies. International Journal of Environment and Pollution, 22(4), 430-440, 2004.

MARTINS, L. D.; ANDRADE, M. F. Ozone formation potentials of volatile organic compounds and ozone sensitivity to their emission in the megacity of São Paulo, Brazil. Water, Air and Soil Pollution, v. 195, p. 201-213, 2008.

MARTINS, L. D.; VASCONCELLOS, P. C.; CARVALHO, L. R. F.; ANDRADE, M. F. Estimated Impact of Biogenic Hydrocarbon Emission on Photochemical Oxidant Formation in São Paulo during two periods of the winters of 1999-2000. Revista Brasileira de Meteorologia, v. 21, n. 2, p. 190-200, 2006.

SÁNCHEZ-CCOYLLO, O. R.; ANDRADE, M. F. The influence of meteorological conditions on the behavior of pollutants 
concentrations in São Paulo, Brazil. Environmental Pollution, 116, 257-263, 2002.

SÁNCHEZ-CCOYLLO, O. R.; YNOUE, R. Y.; MARTINS, L. D.; ANDRADE, M. F. Impacts of Ozone Precursor Limitation and Meteorological Variables on Ozone Concentration in São Paulo, Brazil. Atmospheric Environment, v. 40, p. 552-562, 2006.

SILLMAN, S. The relation between ozone, NOx, and hydrocarbons in urban and polluted rural environments. Atmospheric Environment, 33, 1821-1845, 1999.

SILVA DIAS, M. A. F.; MACHADO, A. J. The role of local circulations in summertime convective development and nocturnal fog in São Paulo, Brazil. Boundary-Layer Meteorology, v. 82, p. 135-157, 1997.

SILVA DIAS, M. A. F.; VIDALE, P. F.; BLANCO, C. M. R. Case study and numerical simulation of the summer regional circulation in São Paulo, Brazil. Boundary-Layer Meteorology, v. 74, p. 371-388, 1995.
ULKE, A. G.; ANDRADE, M. F. Modeling urban air pollution in São Paulo, Brazil: sensitivity of model predicted concentrations to different turbulence parameterizations. Atmospheric Environment, 35, 1747-1763, 2001.

VIVANCO, M. G.; ANDRADE, M. F. Relation between ozone levels and NOx and VOC emissions in the São Paulo Metropolitan Area for an episode of August, 1998. International Journal of Environment and Pollution, v. 35, p. 90-98, 2008.

VUKOVICH, F. M.; SHERWELL, J.; WAYLAND, R. Characteristics of ozone in the Baltimore-Washington area established from 1-hour average observations. Journal of Air and Waste Management Association, 49, 794-803, 1999. 